\title{
Comparable Specimens for Lipid-Laden Macrophage Index: Pediatric Tracheal Aspirate Versus Bronchoalveolar Lavage Fluid
}

\author{
Fergal G. Kavanagh, MB, BCh, BAO'; Karen Watters, MB, BCh, MPH'1,2*; Stephen Kieran, MB, BCh, BAO'; \\ Rachel Rosen, MD ${ }^{3,6}$; Gary Visner, DO ${ }^{4,6}$; Reza Rahbar, DMD, MD ${ }^{1,2}$; Sara O. Vargas, MD
}

\author{
'Department of Otolaryngology and Communication Enhancement, Boston Children's Hospital, Harvard Medical \\ School, Boston, Massachusetts, USA \\ 2Department of Otolaryngology and Head and Neck Surgery, Harvard Medical School, Boston, Massachusetts, USA \\ 3Division of Gastroenterology, Hepatology and Nutrition, Boston Children's Hospital, Harvard Medical School, Boston, \\ Massachusetts, USA \\ ${ }^{4}$ Division of Pulmonary Medicine, Boston Children's Hospital, Harvard Medical School, Boston, Massachusetts, USA \\ ${ }^{5}$ Department of Pathology, Boston Children's Hospital, Harvard Medical School, Boston, Massachusetts, USA \\ ${ }^{6}$ Department of Pediatrics, Harvard Medical School, Boston, Massachusetts, USA
}

\begin{abstract}
Objective: Assessment of lipid-laden macrophages within bronchoalveolar lavage fluid has been proposed to be diagnostically helpful in evaluation of tracheobronchial inflammation. However, measurement requires flexible bronchoscopy with bronchoalveolar lavage, which can be associated with oxygen desaturation and post-operative fever. Quantification of lipid-laden macrophages in tracheal material has not been explored. This study assesses the correlation between lipid-laden macrophage index in tracheal secretions (tLLMI) acquired at the time of direct laryngobronchoscopy (DLB) with the bronchoalveolar LLMI (aLLMI) collected via flexible bronchoscopy.

Methods: Thirty-five pediatric patients undergoing simultaneous DLB and flexible bronchoscopy, at a pediatric tertiary center over a 21-month period as part of a multidisciplinary aerodigestive tract program, underwent both aspiration of tracheal secretions and bronchoalveolar lavage (BAL) with LLMI assessment.

Results: Patients underwent dual upper and lower airway endoscopic assessment for an array of aerodigestive symptoms: chronic cough ( $\mathrm{N}=13$ ), aspiration/recurrent pneumonia ( $N=11)$, recurrent croup $(N=4)$, feeding difficulties $(N=4)$. Mean tracheal LLMI was 38.9 (95\% confidence interval, $C l, 26.4-51.3)$; mean bronchoalveolar LLMI was $45.2(95 \% \mathrm{Cl} 36.0-54.3, \mathrm{P}=0.001)$. There was no statistical difference between the paired groups (Student's paired t-test, $P<0.001)$. Pearson correlation revealed moderate to strong positive correlation between the two groups $(r=0.67, P<0.001)$.

Conclusion: The LLMI in tracheal aspirates collected at the time of DLB correlates with that of aspirates measured directly from the lower airways. This suggests that LLMI from tracheal aspirates have diagnostic potential comparable to those from BAL, and centers which may not have access to BAL could use tracheal aspirates to calculate LLMI in the work-up of patients in whom aspiration is suspected.
\end{abstract}

\section{INTRODUCTION}

Chronic pulmonary aspiration, a condition in which there is intermittent or persistent entry of alimentary contents into the airway, can be responsible for significant pulmonary comorbidity including recurrent aspiration pneumonia, exacerbation of asthma, chronic bronchitis, bronchiectasis, stridor and apnea $[1,2,3]$. This condition occurs when there is a failure of the normal airway protective mechanisms. Chronic aspiration causes clinical, histopathologic and radiological evidence of lung injury and clinicians use multiple modalities to evaluate aspiration including chest x-rays, computed tomography and diagnostic procedures including speech and swallow studies. However, no sensitive or specific test for chronic aspiration has emerged [4]. The difficulty in any of these investigations making a conclusive diagnosis is compounded by the infrequency of the aspiration events and the relatively small volumes aspirated.

The observation of lipid-laden macrophages in the lung as a marker of aspiration was first made in the 1920s [5]. Because lipids present in aspirated gastric contents are phagocytosed by macrophages it has been postulated that analysis of lipid content in macrophages could be a useful indicator of pulmonary aspiration of gastric contents. In bronchoalveolar lavage (BAL) specimens, lipid-laden macrophages are identified cytologically via light microscopy in cytospin preparations stained with oil red-O. The lipid-laden macrophage index (LLMI) was first proposed in the 1980s as a measure to quantitate the extent and intensity of oil red-O staining within macrophages $[6,7]$. It has been put forward as diagnostically helpful in the assessment of aspiration [7].

Although BAL is a well-established and safe technique, it is associated with adverse effects, most commonly post-operative fever. The prevalence of post-bronchoscopy fever is estimated at between 5-68\%, with BAL in particular associated with higher rates of fever [8]. In the pediatric population, the prevalence of fever has been estimated at $48 \%$, and the following factors have been implicated: age <2 years, positive bacterial cultures, abnormal BAL findings [9]. Episodes of oxygen desaturation or apnea have also been reported during BAL procedures, ranging from mild complications, including transient desaturation (14\%) to more prolonged apnea and severe desaturation $(0.6 \%)$ requiring increased mechanical ventilation [10]. The assessment of lipid-laden macrophages in tracheal aspirate specimens has not been extensively studied, with existing studies being focused primarily on reflux aspiration and not performing any comparison of tracheal versus bronchial aspirates [11]. Whilst a correlation between aspiration of tracheal secretions could avoid complications associated with BAL and warrants further investi- 
gation it would not supersede the importance of BAL as the investigation of choice in these patients.

The aim of this study was to assess the correlation between LLMI in tracheal secretions, which were gathered at the time of the DLB with the alveolar LLMI collected via the BAL. To the best of our knowledge and in our most recent literature review no other studies to date have compared LLMI in fluid collected by BAL to that collected by tracheal aspirates. Our hypothesis was that there would be no difference between the aspirates taken at time of DLB and BAL.

\section{METHODS}

The Boston Children's Hospital Institutional Review Board approved this study, and their guidelines were strictly followed. This was a prospective observational study carried out in a tertiary level pediatric hospital over a 21-month period.

\section{Eligibility Criteria}

Patients selected for inclusion were being evaluated as part of a combined aerodigestive disease service for chronic respiratory symptoms managed in conjunction with otolaryngology, pulmonology and gastroenterology. Exclusion criteria were lack of consent to participate by parents or guardians. Patient demographics comprising age, gender, medical history, history of respiratory symptoms and diagnosis were collected. Patients presented as a consecutive series.

The primary outcome measure was comparison of the reference test of LLMI in BAL compared to the index test we proposed by way of tracheal aspirates. Tracheal aspirates were harvested to measure the tracheal LLMI (tLL$\mathrm{MI})$, and BAL specimens were sampled to assay bronchoalveolar LLMI (aLLMI). Both specimens were acquired in the same setting.

\section{Sampling}

All procedures were performed under general anesthesia. Infants were fasted prior to the procedure for 6 hours for milk and solids and 4 hours for water in accordance with the Department of Anesthesiology's fasting guidelines. DLB was performed prior to endotracheal intubation. All tracheal aspirates were performed in this standardized fashion at the time of rigid bronchoscopy under direct endoscopic vision by a single pediatric otolaryngologist (R.R.) using a Lukens trap.

The patients were subsequently endotracheally intubated, and flexible bronchoscopy was performed by a pediatric pulmonologist according to standard clinical practice in Boston Children's Hospital. BAL samples were obtained in the following manner: The bronchoscope was wedged in a sub-segmental bronchus and 1 or 2 aliquots of $1 \mathrm{~mL} / \mathrm{Kg}$ of sterile normal saline at room temperature were instilled and suctioned, and the resulting samples were sent for cytologic analysis.

\section{Cytopathology Analysis}

The BAL and tracheal aspirate fluid was cytospun on slides, air-dried, stained with oil red-O, and counterstained with hematoxylin. For each sample, intracellular lipid levels within 100 macrophages were graded, each with a score of 0 to 4 , where 0 was not opacified and 4 was complete opacification. These scores were then totaled, giving a LLMI score between 0 and 400 . The LLMI level was determined by a certified cytotechnologist in conjunction with an attending pathologist at the same tertiary medical center.

\section{Statistical Analysis}

Statistical analysis was conducted using Analyse-it for Microsoft Excel (version 2.20) software. Pearson correlations were performed to measure the strength of association between continuous variables. The independent t-test was used to compare means of normally distributed data.

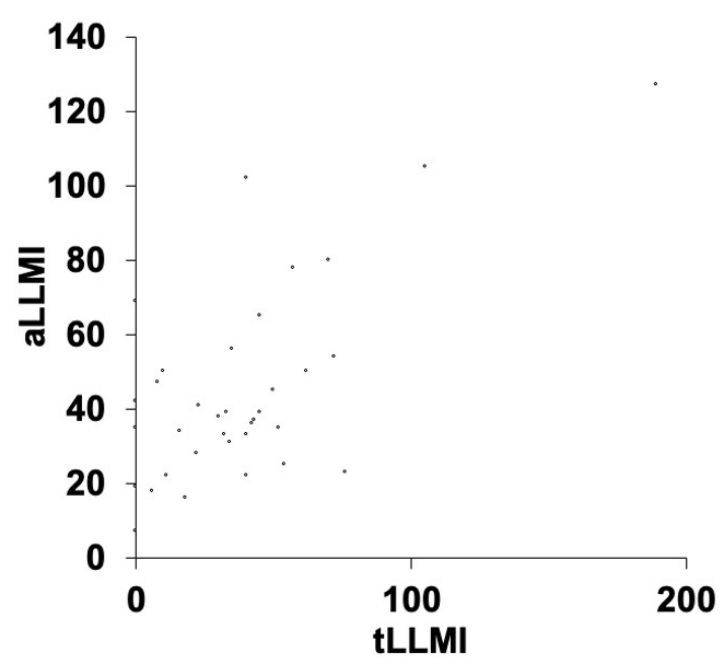

Figure 1. A scatter plot depicting the aLLMI and tLLMI for each patient. The Pearson correlation coefficient $(r)$ for the entire population was 0.67 ( $95 \% \mathrm{Cl} 0.42$ to 0.82 ) representing a strong positive correlation. aLLMI, bronchoalveolar lipid-laden macrophage index; tLLMI, tracheal lipid-laden macrophage index.

\section{RESULTS}

Thirty-five patients who underwent simultaneous DLB and flexible bronchoscopy were included in the study. The cohort comprised 24 males and 11 females, ranging in age from 4 months to 15 years. The mean age at bronchoscopy was 5.4 years (median 4.1 years, range 0.4 years to 15.6 years). Patients underwent dual upper and lower airway assessment for an array of aero-digestive symptoms including chronic cough $(N=13)$, aspiration/recurrent pneumonia $(N=11)$, recurrent croup $(N=4)$, feeding difficulties $(N=4)$, and stridor/noisy breathing $(\mathrm{N}=3)$.

The mean tracheal LLMI was 38.9 (95\% confidence interval, Cl, 26.4-51.3); the mean bronchoalveolar LLMI was 45.2 (95\% Cl 36.0-54.3). There was no statistical difference between the paired groups (Student's paired t-test, $\mathrm{P}$ $<0.001$ ). Pearson correlation revealed a moderate to strong positive correlation between the two groups $(r=0.67,95 \% \mathrm{Cl} 0.43-0.82$, $\mathrm{P}<0.001$, Figure 1$)$. The mean LLMI when patients were grouped by their primary symptom is displayed in Figure 2. The Pearson correlation coefficient was strong for aspiration/recurrent pneumonia, recurrent croup and feeding problems. It was negatively weak for patients presenting with chronic cough $(P=0.0098)$

\section{DISCUSSION}

The exact role of assessment of lipid-laden macrophages in the diagnosis and work up of children with chronic aspiration is controversial even though some hospitals continue to use it as part of their algorithm of chronic lung disease. The goal of this research was not to determine the sensitivity or specificity of LLMI or to further clarify its use in the work up of children with suspected aspiration. Rather the aim of the project was to examine if there is a strong correlation between LLMI when measured contemporaneously in both the lower airways and the trachea.

Our study showed strong correlation between LLMI in tracheal aspirate specimens with LLMI in BAL specimens in a cohort of pediatric patients undergoing assessment of suspected aerodigestive disorders. Aspiration of tracheal 


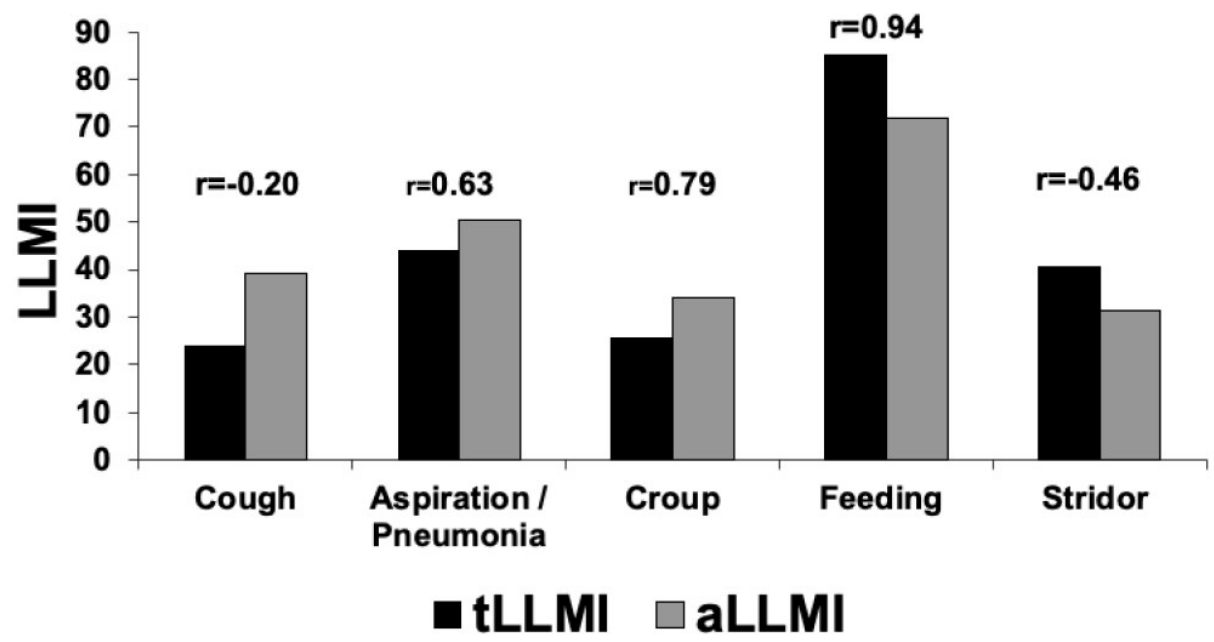

Figure 2. Chart representing the mean tracheal lipid laden macrophage index when patients are grouped by primary symptom. The Pearson correlation coefficient for the tLLMI and aLLMI is depicted above each group. A strong correlation was present for patients presenting with aspiration pneumonia, recurrent croup and feeding disorders. aLLMI, bronchoalveolar lipid-laden macrophage index; tLLMI, tracheal lipid-laden macrophage index.

secretions is a routine part of a rigid bronchoscopy to improve visualization. Therefore, the routine assessment of these secretions is unlikely to be associated with added morbidity or increased operative time and these aspirates may have diagnostic potential. Our study has shown that there is a correlation between tLLMI and aLLMI in a range of pediatric and adolescent conditions including chronic aspiration, recurrent pneumonia, recurrent croup and feeding or oropharyngeal dysphagia with aspiration. We suspect that the correlation between lipid-laden macrophage quantification may extend beyond the pediatric/adolescent age group and into other clinical settings including adult lung disease. Interestingly, recent research has looked at the use of LLMI in vaping-associated pulmonary injury (VAPI), suggesting that elevated LLMI was associated with the diagnosis $[12,13]$. An easier way of acquiring material for the quantification of lipid-laden macrophages may lead to the more widespread use of this test and may help in time help to clarify the use of this investigation, in VAPI and in other respiratory disorders in patients of all ages.

We fully acknowledge, however, that there are clinical situations in which evaluation for additional disease features beyond lipid-laden macrophages may warrant the deeper, broader sampling afforded by BAL. BAL will continue to provide useful information for patients presenting with aerodigestive disease in addition to LLMI index (cultures, presence of eosinophils) and in patients who are undergoing triple scope it is unlikely to lead to the elimination of the BAL portion. Rather the possible utility of this finding may be in centres where coordinated DLB and BAL are not often available or in cases where the otolaryngologist is doing an airway assessment and notes signs of aspiration and may consider this investigation useful in the patient work up.

When the measurement of LLMI via BAL was first introduced it showed promise in identifying chronic aspiration; however, its role is widely disputed. There is significant variation in the sensitivity and specificity of the LLMI for aspiration (57\%-100\% and 57\%-89\% respectively) $[6,7,14,15,16]$. Nonetheless the American Thoracic Society considers elevated LLMI to be 'suggestive but not diagnostic of aspiration' and recommends its continued use [17]. One of the key reasons that the LLMI is not a reliable marker is because of the manner in which it is calculated; with the fat content of 100 consecutive macrophages given a score of 0-4 [7]. LLMI values have a sizeable margin of error, likely due to a lack of consistency in which of any 100 macrophages are encountered as well as due to interobserver variability [18]. Repeated interpretation by different observers shows a sizeable fluctuation in the result. Furthermore, it cannot distinguish between the presence of endogenous and exogenous lipid within the macrophage. Macrophages may become lipid-laden through the phagocytosis of damaged cells in a variety of conditions characterized by alveolar cell injury and LLMI can be elevated independent of aspiration [19].

At our pediatric institution, LLMI have not been routinely provided by pathologists evaluating BAL samples for over five years. Because of its time-consuming nature, the analysis was discontinued in favor of a "gestalt" analysis of absent, mild, moderate, or marked increase in lipid within macrophages, measured by eye without the counting and grading of individual cells. It is expected that tracheal aspirate cytology reporting would likewise be amenable to this interpretive "shortcut," but nevertheless, we consider it helpful to have used the more rigorous measure in the samples studied herein.

The major limitation of this study is that the patients included have complex medical backgrounds and therefore caution must be exercised when interpreting our results and making assumptions in larger groups. In this way there may have been selection bias and the generalizability of results made be limited. The study may also have been subject to information bias given the complicated nature of the interpretation of the LLMI although efforts were made to minimize this. Strengths though include the prospective nature of the study, the multi-disciplinary approach between otolaryngology, pulmonology, gastroenterology and pathology and that the study answers a question not previously addressed in the literature. 


\section{CONCLUSION}

To summarize, our findings show that quantitative measurement of lipid-laden macrophages in tracheal aspirates yields comparable results to measurements in BAL fluid. These preliminary findings suggest the LLMI in tracheal aspirates may have diagnostic potential especially in those patients not undergoing flexible bronchoscopy. However, comparative studies are required in larger populations with wider spectrums of disease (including normal populations), prior to the widespread implementation of this diagnostic tool.

\section{ARTICLE INFORMATION}

*Correspondence: Karen Watters, MB, BCh, MPH, Department of Otolaryngology and Communication Enhancement, Boston Children's Hospital 300 Longwood Avenue Boston, Massachusetts, USA. Email: karen.watters@childrens.harvard.edu

Received: Feb. 25, 2021; Accepted:Jul. 12, 2021; Published: Aug. 20, 202

DOI: 10.24983/scitemed.aohns.2021.00144

Disclosure: This study was presented as a poster at The Triological Society's Annual Meeting at the Combined Otolaryngology Spring Meetings, San Diego, California, United States of America on April 20, 2012.

Funding: The study did not receive any specific grant from funding agencies in the public, commercial, or not-for-profit sectors.

Conflict of Interest: The authors report no financial or other conflict of interest relevant to this article, which is the intellectual property of the authors.

Copyright (c) 2021 The Author(s). This is an open-access article distributed under the terms of the Creative Commons Attribution 4.0 International License (CC-BY).

\section{REFERENCES}

1. Euler AR, Byrne WJ, Ament ME, et al. Recurrent pulmonary disease in children: A complication of gastroesophageal reflux. Pediatrics 1979;63(1):47-51.

2. Contencin $P$, Maurage $C$, Ployet MJ, Seid $A B$, Sinaasappel M. Gastroesophageal reflux and ENT disorders in childhood. Int J Pediatr Otorhinolaryngol 1995;32 Suppl:S135-144.
3. Mansfield LE, Stein MR. Gastroesophageal reflux and asthma: A possible reflex mechanism. Ann Allergy 1978;41(4):224-226.

4. Boesch RP, Daines C, Willging JP, et al. Advances in the diagnosis and management of chronic pulmonary aspiration in children. Eur Respir J 2006;28(4):847-861.

5. Pinkerton H. The reaction to oils and fats in the lung. Arch Pathol 1928;5:380-401.

6. Colombo JL, Hallberg TK. Recurrent aspiration in children: Lipid-laden alveolar macrophage quantitation. Pediatr Pulmonol 1987;3(2):86-89.

7. Corwin RW, Irwin RS. The lipid-laden alveolar macrophage as a marker of aspiration in parenchymal lung disease. Am Rev Respir Dis 1985;132(3):576-581.

8. Rubinstein-Aguñín P. Infectious complications following bronchoscopy: Does sedation play a role? J Lung Pulm Respir Res 2018;5(4):112-118.

9. Picard E, Schwartz S, Goldberg S, Glick T, Villa Y, Kerem E. A prospective study of fever and bacteremia after flexible fiberoptic bronchoscopy in children. Chest 2000;117(2):573-577.

10. Efrati O, Sadeh-GornikU, Modan-Moses D, et al. Flexible bronchoscopy and bronchoalveolar lavage in pediatric patients with lung disease. Pediatr Crit Care Med 2009;10(1):80-84.

11. Krishnan U, Mitchell JD, Tobias V, Day AS, Bohane TD. Fat laden macrophages in tracheal aspirates as a marker of reflux aspiration: A negative report.J Pediatr Gastroenterol Nutr 2002;35(3):309-313.

12. Maddock SD, Cirulis MM, Callahan SJ, et al. Pulmonary lipid-laden macrophages and vaping NEnglJ Med 2019;381(15):1488-1489.

13. Itoh $\mathrm{M}$, Aoshiba $\mathrm{K}$, Herai $\mathrm{Y}$, Nakamura $\mathrm{H}$, Takemura T. Lung injury associated with electronic cigarettes inhalation diagnosed by transbronchial lung biopsy. Respirol Case Rep 2018;6(1):e00282

14. Ahrens P, Noll C, Kitz R, Willigens P, Zielen S, Hofmann D. Lipid-laden alveolar macrophages (LLAM): A useful marker of silent aspiration in children. Pediatr Pulmonol 1999;28(2):83-88.

15. Yang YJ, Steele CT, Anbar RD, SinacoriJT, Powers CN. Quantitation of lipid-laden macrophages in evaluation of lower airway cytology specimens from pediatric patients. Diagn Cytopathol 2001;24(2):98-103.

16. Ding Y, Simpson PM, Schellhase DE, Tryka AF, Ding L, Parham DM. Limited reliability of lipidladen macrophage index restricts its use as a test for pulmonary aspiration: Comparison with a simple semiquantitative assay. Pediatr Dev Pathol 2002;5(6):551-558.

17. Kurland G, Deterding RR, Hagood JS, et al. An official American Thoracic Society clinical practice guideline: Classification, evaluation, and management of childhood interstitia lung disease in infancy. Am J Respir Crit Care Med 2013;188(3):376-394.

18. Reid-Nicholson M, Kulkarni R, Adeagbo B, Looney S, CrosbyJ. Interobserver and intraobserver variability in the calculation of the lipid-laden macrophage index: Implications for its use in the evaluation of aspiration in children. Diagn Cytopatho/ 2010;38(12):861-865.

19. Knauer-Fischer S, Ratjen F. Lipid-laden macrophages in bronchoalveolar lavage fluid as a marker for pulmonary aspiration. Pediatr Pulmonol 1999;27(6):419-422. 